\title{
MMP-9 in Myocardial Fibrosis and Structural Remodeling in Rheumatic Heart Disease Patients with Atrial Fibrillation: A Single-center Prospective Case-control Study
}

Shuguo Yuan ( $419090549 @ q q . c o m$ )

Yue Bei People's Hospital

\section{Wan Li}

Yue Bei People's Hospital

Liangqiu Tang

Yue Bei People's Hospital

Chengquan Liao

Yue Bei People's Hospital

Shaochun Ma

Yue Bei People's Hospital

Fengping $\mathrm{He}$

Yue Bei People's Hospital

Wenmao Fan

Yue Bei People's Hospital

Shebing Zhang

Yue Bei People's Hospital

Zhanzhong Ma

Yue Bei People's Hospital

Shuangquan Gao

Yue Bei People's Hospital

Yun Deng

Yue Bei People's Hospital

Qiuxiao Yuan

Yue Bei People's Hospital

\section{Research Article}

Keywords: atrial fibrillation, matrix metalloproteinase-9, collagen volume fraction, left atrial diameter, cardiac structural remodeling 
Posted Date: January 18th, 2021

DOl: https://doi.org/10.21203/rs.3.rs-140322/v1

License: (c) (i) This work is licensed under a Creative Commons Attribution 4.0 International License. Read Full License 


\section{Abstract}

Background: Atrial fibrillation (AF) is a common arrhythmia that induces disability or death; however, the underlying pathogenesis is yet unclear. This study aimed to investigate the expression of matrix metalloproteinase-9 (MMP-9) in rheumatic valvular heart disease (RVHD) and its value in predicting AF.

Methods: In this single-center, prospective case-control study, 30 patients who received valve replacement for the treatment of RVHD in the Cardiac and Major Vascular Department, Yue Bei People's Hospital between August 2012 and January 2015 were included in this study. The patients were categorized equally into two groups according to the electrocardiogram characteristics: sinus rhythm (SR) group and chronic atrial fibrillation (AF) group. In patients hospitalized for RVHD, the fasting serum was collected on the following day in the morning, and the tissues of right auricle were collected during the operation. Hematoxylin and eosin staining and Masson staining were performed to assess the pathological structures of myocardial tissues and changes in collagen fibers. Enzyme-linked immunosorbent assay was used to measure the level of MMP-9 in serum, and the distribution of the protein in myocardial tissues was assessed by immunohistochemistry. Echocardiography was performed before the surgery to measure the left atrial diameter and ejection fraction.

Results: The MMP-9 expression was significantly higher in the AF group than the SR group (4.2281 \pm $0.9165 \mathrm{ng} / \mathrm{mg} v s .2 .7613 \pm 1.2166 \mathrm{ng} / \mathrm{mg}, p<0.05)$. The MMP-9 levels were positively associated with left atrial diameter (LAD) $(p<0.01)$ and collagen volume fraction (CVF) $(p<0.05)$.

Conclusions: MMP-9 expression is elevated in AF patients and could be one of the major factors involved in the remodeling of atrial structures in AF. MMP-9 influences the metabolism of collagen and promotes the fibrosis of the myocardium, participates in cardiac structural remodeling, and plays a role in the onset and maintenance of AF.

\section{Background}

Rheumatic valvular heart disease (RVHD) is mainly characterized by valvular disorders induced by acute rheumatic carditis. RVHD is a common organic valvular heart disease, which accounts for about $80-85 \%$ of all valvular heart diseases. Atrial fibrillation (AF) is a super-ventricular tachyarrhythmia characterized by rapid, unordered atrial electrical activities that induce cardiac failure, arterial embolism, and cerebral stroke, and the rates of disability and lethality induced by AF are high [1-3]. Typically, AF is secondary to RVHD. Previous studies have reported that AF patients with RVHD exhibit atrial remodeling, manifested as fibrosis of atrial tissues [4]. Myocardial fibrosis (MF) refers to the imbalance of the synthesis and degradation of myocardial extracellular matrix (ECM) after myocardial injuries that lead to increased aggregation of extracellular matrix, proliferation of fibroblast, and differentiation of fibroblasts to myofibroblast (MFB), which disturb the continuity of myocardial fiber bundles, damage myocardiumfibroblast interactions, and induce myocardial remodeling. Therefore, MF is a critical structural basis for 
ectocardia, triggered activity, or reentry, which induce the formation of AF-susceptible matrix and promote the development and progression of AF [4].

Previous studies have shown that matrix metalloproteinases (MMPs), such as MMP-9, are vital components of myocardial tissues that participate in interstitial fibrosis and degrade the matrix, regulate stomatin formation, induce bioactive factor release by ECM, disturb the balance of synthesis and degradation of ECM, and thus lead to hyperplasia and aggregation of ECM, consequently promoting ECM remodeling [5] and inducing MF. Previous studies have demonstrated that the expression of microRNA223 and MMP-9 is significantly increased in RVHD patients with AF; however, the underlying mechanisms are yet unclear. We hypothesized that MMP-9 regulates the abnormal synthesis and degradation of ECM to induce structural remodeling of ECM, which participates in MF and the structural remodeling of cardiac tissues [6]. This study aimed to assess the expression of MMP-9 and collagen volume fraction (CVF) in RVHD patients with AF, explore the roles of MMP-9, CVF, and left atrial diameter (LAD) in the early diagnosis of atrial remodeling and MF in RVHD patients, investigate the underlying mechanisms, and uncover the predictive effects on the the initiation and maintenance of AF.

\section{Methods}

\section{Patients}

In this single-center, prospective case-control study, 30 patients who underwent valve replacement for the treatment of RVHD in the Cardiac and Major Vascular Department, Yue Bei People's Hospital between August 2012 and January 2015, were included. The patients were categorized equally into two groups according to the electrocardiogram (ECG) characteristics as follows: sinus rhythm (SR) group and chronic AF group.

\section{Inclusion and exclusion criteria}

The inclusion criteria of the patients were as follows: 1) RVHD patients with indications for cardiac valve replacement; AF patients also met the ECG diagnostic criteria; 2) NYHA grade of heart was grade II-III; 3) Vital signs and the disease condition was stable; 4) Clear consciousness, volunteered to participate in this study, and cooperated with the treatments. The exclusion criteria were as follows: 1) Other types of heart diseases, such as myocardial infarction and acute myocarditis, within the last month; 2) Complications such as a malignant tumor, chronic obstructive pulmonary disease (COPD), and uremia; 3 ) Cardiac function of grade IV.

This study was approved by the Ethics Committee of the Yue Bei People's Hospital. The serum and tissues of the right auricle were collected during hospitalization. The collection of serum and tissues were performed after consent from the families. Written informed consent was also obtained. 


\section{Diagnosis of AF}

For the diagnosis of AF, patients must meet at least one of the following criteria: 1) current ECG showed $\mathrm{AF}$ and 2) patients had been diagnosed with AF before.

According to the Guidelines for the Management of Atrial Fibrillation issued by the European Society of Cardiology (ESC) in 2010, AF was classified into the following five types [7]: 1) first diagnosed AF: The patients are diagnosed with AF for the first time; 2) paroxysmal AF: the AF is self-terminating, usually within $48 \mathrm{~h}$ and does not persist for more than 7 days. If the AF persisted for $>48 \mathrm{~h}$, the likelihood of spontaneous conversion is low, and anticoagulation must be considered; 3 ) persistent AF: the AF episode either lasts for $>7$ days or requires termination by drug therapy or direct current cardioversion; 4) longstanding persistent $\mathrm{AF}$ : the $\mathrm{AF}$ lasts for $>1$ year and requires rhythm control strategy; 5) permanent AF: both drug therapy and direct current cardioversion failed, and the presence of AF is accepted by patients and physicians.

According to the New York Heart Disease Association (NYHA), the cardiac functions were graded as follows [8]: 1) grade I, physical activities are not limited, and general physical activities do not induce excessive weakness, palpitation, or shortness of breath; 2) grade II, physical activities are slightly limited, and no discomfort is reported during rest, while several daily activities induces weakness, palpitation, or shortness of breath; 3 ) grade III, physical activities are evidently limited, no discomfort is reported during rest, while the load less than daily activities induce weakness, palpitation, or shortness of breath; 4) could not perform any physical activities without symptoms; symptoms could appear during rest, and any physical activities could increase the discomfort.

After hospitalization, patients were also excluded if the medical history, physical examination, and laboratory examination results ruled out malignant tumors. Also, patients with the cardiac function of grade IV were excluded.

\section{Equipment and materials}

Modified Masson tri-staining dye was from the Maiwei Biotechnology Co. Ltd (Xiamen, China). Rabbitanti-human anti-MMP-9 antibody, working solution of horseradish peroxidase (HRP)-labeled streptavidin, and enzyme-linked immunosorbent assay (ELISA) kits for human MMP-9/gelatinase-B were purchased from the Zhongshanjinqiao Biotechnology Co. Ltd (Beijing, China). Goat-anti-rabbit IgG-HRP antibody was purchased from Bioworld Technology (USA). Other reagents were obtained from the Department of Laboratory Medicine, Yue Bei People's Hospital.

\section{Sample collection}


A volume of $5 \mathrm{~mL}$ venous whole blood was collected in an RNA-free dry vacuum tube by centrifugation at $3000 \mathrm{rpm}$ for $20 \mathrm{~min}$ at room temperature. The serum was transferred into a nuclease-free Eppendorf tube and stored at $-80^{\circ} \mathrm{C}$ until further use.

The cardiac tissues of all patients were harvested by a chief surgeon who had worked in the Cardiothoracic Surgery Department for over 20 years. All the pathological examinations were performed by an attending pathologist with 10 years of experience. During the operation, an equivalent of $200 \mathrm{mg}$ myocardial tissues of the right auricle was harvested, rinsed by normal saline to remove blood, and the adipose tissues removed. Then, the myocardial tissues were placed in liquid nitrogen until use. In addition, a part of myocardial tissues was fixed with $10 \%$ neutral formalin liquid, and embedded in paraffin.

\section{Hematoxylin-eosin (H\&E) staining of myocardial tissues}

The myocardial tissues were routinely embedded, sliced, dewaxed by xylene, dehydrated by gradient ethanol, washed, and stained with hematoxylin. Then, the tissues were washed again, developed in a hydrochloric acid ethanol solution, counterstained by eosin, washed, dehydrated, cleared, and mounted by neutral balsam.

\section{Masson's staining of myocardial tissues}

After Masson staining, the myocardial tissues were examined by light microscopy $(\times 400)$ to assess the contents and distribution of collagen fibers. For each sample, images of five random visual fields were captured. The Image Pro Plus, 6.0 image analysis system, was used for the analysis of the images and assess the percentage of collagen fibers in the overall area of myocardial tissues.

\section{Immunohistochemistry of myocardial tissues}

The myocardial tissues were embedded in paraffin and sliced, followed by dewaxing and rehydration. Then, the slices were incubated with $3 \% \mathrm{H}_{2} \mathrm{O}_{2}$ for 5-10 min at room temperature, washed with distilled water, and immersed in phosphate-buffered saline (PBS) for $5 \mathrm{~min}$. Subsequently, 5-10\% normal goat serum (diluted in PBS) was used to block the sections for $10 \mathrm{~min}$ at room temperature and incubated with rabbit-anti-human MMP-9 antibody at $37^{\circ} \mathrm{C}$ for $2 \mathrm{~h}$ and then with HRP-labeled streptavidin at room temperature for $30 \mathrm{~min}$. Finally, the slices were washed with PBS and developed using Diaminobenzidine (DAB) for 3-15 min, rinsed under running water, counterstained, dehydrated, cleared, and mounted.

\section{Measuring serum MMP-9 level with ELISA}


Fasting peripheral venous blood was withdrawn in the morning when the patients were conscious. ELISA kits (Boster, Wuhan, China) were used to measure the MMP-9 level by the same investigator from the Department of Laboratory Medicine, according to the manufacturer's instructions.

\section{Measurement of LAD and ejection fraction (EF)}

Echocardiography was performed by an attending physician at the Department of Sonography before the operation, and the LAD and EF were measured.

\section{Statistical analysis}

SPSS19.0 software was used for the statistical analysis. Quantitative data were described using means and standard deviations. Unpaired Student's $t$-test was conducted for the comparisons between two groups of continuous data. Qualitative data were compared using $\chi^{2}$ test. Pearson's linear correlation analysis was performed for the correlation test. Multivariate logistic regression analysis was performed to investigate the association between general characteristics, including age, sex, smoking history, LAD, systolic blood pressure (SBP), diastolic blood pressure (DBP), blood lipid, creatinine (Cr), uric acid (UA), and blood glucose with MMP-9 levels, and further assess the effect sizes. $p<0.05$ indicated statistical significance.

\section{Results}

\section{Clinical characteristics of patients}

The LAD, SBP, and serum MMP-9 levels were significantly higher in the AF group than the SR group ( $p<$ $0.05)$. However, the age, smoking history, blood lipid, blood glucose, $\mathrm{Cr}, \mathrm{UA}$, blood urea nitrogen (BUN), and SBP did not differ significantly between the two groups $(p>0.05)$ (Table 1). After the adjustment of sex, $L A D$, and SBP by logistic regression, the MMP-9 level was still significantly different between the two groups $(p<0.05$, odds ratio $[\mathrm{OR}]=5.066)$ (Table 2$)$. 
Table 1

Comparisons of baseline characteristics between the two groups $(X \pm S)$

\begin{tabular}{|llll|}
\hline Parameter & SR group $(\mathbf{n}=15)$ & AF group $(\mathbf{n}=15)$ & P-value \\
\hline Age (years) & $52.92 \pm 11.51$ & $49.75 \pm 10.14$ & 0.93 \\
\hline Sex & $5 / 10$ & $12 / 3$ & 0.002 \\
\hline Smoking history & $2 / 13$ & $2 / 13$ & 0.759 \\
\hline LAD (mm) & $36.67 \pm 8.07$ & $58.00 \pm 14.75$ & $<0.01$ \\
\hline Cardiothoracic ratio (\%) & $0.560 \pm 0.055$ & $0.63 \pm 0.049$ & $<0.01$ \\
\hline EF (\%) & $0.58 \pm 0.096$ & $0.55 \pm 0.075$ & 0.752 \\
\hline SBP (mmHg) & $125.47 \pm 16.168$ & $109.53 \pm 15.941$ & 0.323 \\
\hline DBP (mmHg) & $77.40 \pm 11.016$ & $70.938 \pm 11.156$ & 0.021 \\
\hline Alanine transaminase (u/L) & $34.31 \pm 25.012$ & $22.84 \pm 15.382$ & 0.096 \\
\hline Cr ( $\mu$ mol/L) & $110.867 \pm 42.189$ & $109.187 \pm 54.88$ & 0.448 \\
\hline Blood glucose (mmol/L) & $5.752 \pm 1.972$ & $6.015 \pm 2.606$ & 0.198 \\
\hline Total cholesterol (mmol/L) & $3.997 \pm 0.610$ & $3.806 \pm 0.285$ & 0.505 \\
\hline Triglyceride (mmol/L) & $0.921 \pm 0.405$ & $1.060 \pm 0.260$ & 0.288 \\
\hline High-density lipoprotein cholesterol (mmol/L) & $1.231 \pm 0.322$ & $1.109 \pm 0.256$ & 0.183 \\
\hline Low-density lipoprotein cholesterol (mmol/L) & $2.242 \pm 0.622$ & $2.346 \pm 0.357$ & 0.716 \\
\hline UA ( $\mu$ mol/L) & $327.53 \pm 127.513$ & $360.87 \pm 117.249$ & 0.504 \\
\hline BUN (mmol/L) & $6.995 \pm 2.677$ & $6.184 \pm 1.887$ & 0.062 \\
\hline MMP-9 (ng/mL) & $2.7049 \pm 0.9533$ & $4.0812 \pm 1.131$ & $<0.01$ \\
\hline CVF (\%) & $14.6 \pm 0.05$ & $8.6 \pm 0.03$ & $<0.01$ \\
\hline SR: sinus rhythm; AF: atrial fibrillation; LAD: left atrial diameter; EF: ejection fraction; SBP: systolic \\
\hline blood pressure; DBP: diastolic blood pressure; Cr: creatinine; UA: uric acid; BUN: blood urea nitrogen; \\
\hline MMP-9: matrix metalloproteinase-9; CVF: collagen volume fraction. & & \\
\hline
\end{tabular}

Table 2

Comparison of MMP-9 between the two groups by adjusting age, sex, LAD, and SBP by logistic analysis

\begin{tabular}{|lcccccc|}
\hline Independent variable & B value & SE & $\chi^{2}$ value & OR & $95 \% \mathrm{Cl}$ & $P$ \\
\hline MMP-9 & 1.623 & 0.609 & 7.099 & 5.066 & $1.536-16.712$ & 0.008 \\
\hline MMP-9: matrix metalloproteinase-9; LAD: left atrial diameter; SBP: systolic blood pressure. \\
\hline
\end{tabular}




\section{Pathological change in the myocardial fibers from the atrial myocardial tissues}

Light microscopy of the H\&E stained slices showed that the myocardium was larger, myocardial bundles were thicker, cellular nuclei were larger, the arrangement of myocardium was disturbed, and myocardial matrix was increased in the AF group as compared to the myocardial tissues in the SR group (Fig. 1). Light microscopy of the Masson-stained slices showed that myocardial interstitial fibrosis was severe in the AF group, which led to the multidirectional arrangement and size of the myocardium. The MF of the right atrium is shown in Fig. 2. The CVF was significantly different between the AF and SR groups (14.6 \pm $0.05 \%$ vs. $8.0 \pm 0.03 \%, p<0.01)$.

\section{Expression of MMP-9 protein in myocardial tissues}

The expression of MMP-9-positive cells in atrial myocardial tissues was substantially higher in the AF group than the SR group. Large area distribution of brown-yellow staining was found in the cytoplasm of the myocardium in the AF group, while only dispersed distribution was detected in the SR group (Fig. 3).

\section{Expression of MMP-9 in serum}

The serum MMP-9 levels in the AF and SR groups are shown in Table 1. Compared to the SR group, the MMP-9 level in the AF group was significantly higher (4.2281 \pm 0.9165 vs. $2.7613 \pm 1.2166, p<0.05)$ (Fig. 4). In addition, the CVF in the AF group was significantly higher than in the SR group ( $14.6 \pm 0.05 \%$ vs. $(8.0 \pm 0.03 \%, p<0.05)$ (Fig. 5). The MMP-9 protein levels were positively associated with LAD $(r=0.894, p$ $<0.01)$ (Fig. 6) and CVF $(r=0.406, p<0.05)$.

\section{Discussion}

The natural course of AF suggests it is a progressive disease, which develops from paroxysmal AF to persistent AF. With advanced age, the incidence of AF also increases accordingly. The long-term blood stasis in the atrium and dysfunction of vascular endothelium during AF episode could cause the hypercoagulable state and induce embolism, which in turn, induces cerebral embolism and systemic circulation embolism. These led to various cardiovascular complications, including cerebral stroke, myocardial infarction, arteriosclerosis, and heart failure $[9,10]$. Therefore, the present study aimed to explore the atrial remodeling and levels of fibrosis-related factors, as well as the clinical significances in AF patients, which provided scientific evidence for the early diagnosis and treatment of AF.

Atrial remodeling is one of the most important mechanisms involved in the development of AF, which is manifested as structural remodeling, including structural changes by fibrosis of ECM or cellular apoptosis $[11,12]$. The ECM of the myocardium not only supported and protected the complete myocardial structures but also played important roles in the transmission of intercellular signals. Currently, it is 
acknowledged that the fibrosis of the atrial matrix is the underlying pathogenesis of AF development [13, 14]. However, several mechanisms are involved in the MF during AF, which include B1 signals of myocardial fibrosis and myoblasts, MMPs, and tissue inhibitors of MMPs. Among these, MMPs are a group of critical enzymes involved in the participation of myocardial tissues in interstitial fibrosis, which regulates the synthesis and degradation of ECM to promote ECM remodeling [12].

MMPs constitute a group of proteinases that could hydrolyze various components in ECM (such as collagen, fibronectin, and proteoglycan). The activities of MMPs rely on metal ions, including $\mathrm{Zn}^{2+}, \mathrm{Ca}^{2+}$, and $\mathrm{Mg}^{2+}$ [15]. MMP-9 is one of the most important enzymes among MMPs and is also reported as gelatinase B or type IV collagen in some previous studies. MMP-9 is secreted as zymogen to extracellular spaces, which is activated by the removal of the propeptide domain through hydrolysis mediated by fibrinolysin-dependent and -independent pathways, followed by selective binding to the components of ECM. MMP-9 is critical for ECM remodeling and plays a critical role in the prognosis of various diseases, including coronary heart disease, valvular heart disease, AF, ventricular remodeling after myocardial infarction, and cardiovascular diseases [5].

The findings of this study demonstrated that compared to the SR group, H\&E staining in the AF group showed enlargement of the myocardium, thickening of myocardial bundles, evident increase in cellular nuclei, disarrangement of the myocardium, and a substantial increase in the myocardial matrix. The Masson staining also demonstrated that myocardial interstitial fibrosis was severe in the AF group, which led to multidirectional arrangement and sizes of the myocardium. These findings demonstrated that the myocardial matrix and collagen fibers in the AF group increased substantially. In addition, the AF group also showed substantial fibrosis of atrial myocardium and ECM, as well as cell apoptosis, which further induced structural changes that led to atrial structural remodeling. These findings were in agreement with the pathogenesis of the development and progression of AF that the structural remodeling of atrium provides susceptible matrix for the development and progression of AF, which is an important structural basis for triggered activity and reentry of ectocardia. These findings were in agreement with those reported in previous studies.

The prominent manifestation of atrial structural remodeling is the fibrosis of atrial matrix, which increases the susceptibility of AF [16-18]. MMP-9 and CVF play critical roles in atrial fibrosis. CVF directly reflects the contents of myocardial collagen fibers and indirectly reflect the severity of myocardial fibrosis. In this study, CVF was selected as an indicator reflecting MF for the investigation of the correlation between MMP-9 and CVF. Compared to the SR group, the MMP-9 and CVF levels in the myocardial tissues in AF group were significantly higher $(p<0.01)$. In addition, the expression of MMP-9 was positively associated with CVF levels $(r=0.406, p<0.05)$. The increased CVF levels in atrial myocardium in AF group suggested a substantial increase in collagen fibers and severity of atrial MF. The positive association between MMP-9 and CVF levels indicated that MMP-9 induces the abnormal metabolism of myocardial collagen by promoting the degradation of normal collagen, increase the abnormal myocardial matrix components, increase CVF, and induce remodeling of atrial myocardial structures. This results in a susceptible environment for AF and consequently promoting the development 
and maintenance of AF $[19,20]$. These finding were in agreement with the results of previous studies. Xu et al. [5] reported that in 53 patients who received cardiac transplantation for dilated cardiomyopathy or end-stage heart failure, the level of MMP-9 expression in the atrial myocardium and type I collagen volume fraction were significantly higher in the AF group than the SR group. These findings indicated that MMP-9 could be used as a marker reflecting atrial remodeling in AF patients [21], while regulating the extracellular collagen matrix that could be a potential treatment target for AF [5].

The present study also investigated the linear correlation between MMP-9 and LAD by Pearson's correlation analysis. The findings demonstrated that MMP-9 expression was positively associated with LAD $(r=0.894, p<0.01)$, indicating that higher the MMP-9 levels, larger the LAD. Multivariant linear regression was adopted to control the influence of other risk factors, including age, SBP, DBP, triglyceride, and UA while the data showed that MMP-9 expression was positively associated with LAD $(p<0.01)$. We speculated that the activation and increased expression of MMP-9 promotes the collagen metabolism in atrial tissues, which leads to overdegradation of normal collagen and the consequent absence of intermyocardial connecting structures. Therefore, MMP-9 activation and overexpression is associated with the enlargement of the atrium. The enlarged atrium could also pull myocardial fibroblasts and induce the expression of MMP-9. Consistent with these findings, Yukiko et al. also showed that the MMP-9 expression was significantly higher in the AF group than the RSR group, while the activity of gelatinase was positively associated with $\operatorname{LAD}(r=0.766, p<0.05)$. These findings suggested that the MMP-9 expression was increased in AF tissues, which could promote the atrial and ventricular remodeling and dilation during AF [22]. Thus, the increased MMP-9 expression promotes the degradation of normal collagen and leads to the absence of connecting structures among normal myocardial tissues, which consequently results in the enlargement of atrial structure and increased LAD. Ke et al. [23] also reported that 75 patients with rheumatic heart disease who underwent valve replacement, the mRNA and protein level of MMP-2, MMP-9 are significantly elevated in the right atrial tissues in AF patients, which were positively associated with $L A D$, and the duration of AF lasted. The overactivation and increased expression of MMP-9 during AF leads to ECM degradation and absence of intermyocardial connecting structures, while the atrial fibrosis could increase the length of the myocardium by increasing the atrial pressure, which consequently thinned the atrial wall and led to the enlargement of atrium. In addition, the synthesis of abnormal collagen protein and connective tissues could lead to the filling of normal ECM lacking intermyocardial spaces by other connective tissues and fibrotic tissues with abnormal structures. Such changes further worsened the MF gradually, led to matrix susceptibility to AF occurrence, and promoted the development of AF $[6,24,25]$. In summary, MMP-9 and atrial dilation are associated with atrial structural remodeling during AF. The change in myocardial matrix and collagen fiber contents to form the AF-susceptible matrix, consequently promoted MF as an underlying mechanism for AF.

The findings of this study also showed that MMP-9 was negatively associated with SBP, which is associated with the varied degree of collagen fiber hyperplasia in myocardial matrix and around the blood vessels during AF. Such changes increase the myocardial stiffness and reduce myocardial compliance. In addition, the abnormal hyperplasia of myocardial matrix reduces the capillary bed in the matrix, which leads to the death of myocardium by chronic hypoxia or cellular apoptosis. Such 
decompensation leads to cardiac electrophysiological abnormalities and a substantial reduction in cardiac systolic and diastolic functions. Furthermore, AF induces blood ejection disorders of the heart and reduces the ventricular diastole phase, which consequently results in sa substantial reduction of stroke volume of the heart, and in turn, reduce the SBP.

The pathogenesis of AF is complex. However, the present study only focused on rheumatic heart disease using a sample size. In addition, this study only explored the MMP-9 expression from the aspect of atrial fibrosis, while the exact underlying mechanisms were not investigated further. Thus, additional studies are needed to investigate the exact mechanisms underlying specific signaling pathways, and explore the effects of related upstream genes (by gene knockout or inhibition) on the expression of MMP-9 in animal models.

In summary, measuring MMP-9 level and LAD in patients with rheumatic heart disease helps in assessing the CVF in myocardial tissues, which replaces the invasive myocardial biopsy to indirectly reflect the severity of MF and serve as an early diagnostic marker for myocardial tissue remodeling and MF, as well as predict the occurrence of AF. In addition, inhibiting the expression or activation of MMP-9 from regulating collagen metabolism inhibits MF and improves myocardial remodeling. In addition, measuring MMP-9 expression and LAD helps in assessing the treatment efficacy and predicting prognosis. The present study demonstrated the value of MMP-9 as a biomarker for the early diagnosis of myocardial remodeling and prediction of AF risk, which could be widely applied in clinical practices. These findings provided a new alternative for the diagnosis and treatment of AF.

\section{Abbreviations}

AF: Atrial fibrillation

MMP-9: matrix metalloproteinase-9

RVHD: rheumatic valvular heart disease

SR: sinus rhythm

AF: atrial fibrillation

CVF: collagen volume fraction

MF: myocardial fibrosis

ECM: extracellular matrix

MFB: myofibroblast

MMPs: matrix metalloproteinases 
LAD: left atrial diameter

ECG: electrocardiogram

COPD: chronic obstructive pulmonary disease

ESC: European Society of Cardiology

NYHA: New York Heart Disease Association

HRP: horseradish peroxidase

ELISA: enzyme-linked immunosorbent assay

H\&E: Hematoxylin-eosin

PBS: phosphate-buffered saline

DAB: Diaminobenzidine

EF: ejection fraction

SBP: systolic blood pressure

DBP: diastolic blood pressure

Cr. creatinine

UA: uric acid

BUN: blood urea nitrogen

OR: odds ratio

\section{Declarations}

\section{Ethics approval and consent to participate}

All methods were carried out in accordance with relevant guidelines and regulations. This study was approved by the Ethics Committee of the Yue Bei People's Hospital. The serum and tissues of the right auricle were collected during hospitalization. The collection of serum and tissues were performed after written informed consent from the families.

\section{Consent for publication}


Written informed consent was obtained from the patients or their legal representative for publication of this article and any accompanying images. A copy of the written consent is available for review by the Editor of this journal.

\section{Availability of data and materials}

All data generated or analyzed during this study are included in this published article.

\section{Competing interests}

The authors declare that they have no financial and non-financial competing interests.

\section{Funding}

This work was supported by the Natural Science Foundation of Guangdong Province [grant number 915100901000039], the Shaoguan City Science and Technology Bureau Project [grant number 2013CX/K11] and the Shaoguan City Health Family Planning Research Project [grant number Y19013].

\section{Authors' contributions}

Shuguo Yuan and Wan Li Qiuxiao Yuan carried out the studies, participated in collecting data, and drafted the manuscript. Liangqiu Tang, Chengquan Liao, Shaochun Ma, Fengping He, Wenmao Fan, Shebing Zhang, Zhanzhong Ma, Shuangquan Gao, Yun Deng and Qiuxiao Yuan performed the statistical analysis, prepared figures and participated in its design. Shuguo Yuan and Wan Li helped to review and edit the manuscript. All authors read and approved the final manuscript.

\section{Acknowledgements}

Not applicable.

\section{References}

1. Pistoia F, Sacco S, Tiseo C, Degan D, Ornello R, Carolei A. The Epidemiology of Atrial Fibrillation and Stroke. Cardiol Clin. 2016;34(2):255-68.

2. Nanda A, Kabra R. Racial Differences in Atrial Fibrillation Epidemiology, Management, and Outcomes. Curr Treat Options Cardiovasc Med. 2019;21(12):85.

3. Cardiology CSo, Engineering RsoCSoB, Association PCoE-bMoCMD, Society C-cDPCoCG. Expert consensus on emergency treatment of arrhythmia. Chinese Journal of Cardiology. 2013;41(5):363- 
76.

4. Krueger MW, Rhode KS, O'Neill MD, Rinaldi CA, Gill J, Razavi R, Seemann G, Doessel O. Patientspecific modeling of atrial fibrosis increases the accuracy of sinus rhythm simulations and may explain maintenance of atrial fibrillation. J Electrocardiol. 2014;47(3):324-8.

5. Lewkowicz J, Knapp M, Tankiewicz-Kwedlo A, Sawicki R, Kamińska M, Waszkiewicz E, Musiał WJ. MMP-9 in atrial remodeling in patients with atrial fibrillation. Ann Cardiol Angeiol (Paris). 2015;64(4):285-91.

6. Moe GW, Laurent G, Doumanovskaia L, Konig A, Hu X, Dorian P. Matrix metalloproteinase inhibition attenuates atrial remodeling and vulnerability to atrial fibrillation in a canine model of heart failure. $\mathrm{J}$ Card Fail. 2008;14(9):768-76.

7. Camm AJ, Kirchhof P, Lip GY, Schotten U, Savelieva I, Ernst S, Van Gelder IC, Al-Attar N, Hindricks G, Prendergast $B$, et al. Guidelines for the management of atrial fibrillation: the Task Force for the Management of Atrial Fibrillation of the European Society of Cardiology (ESC). Europace. 2010;12(10):1360-420.

8. Metra M, Teerlink JR. Heart failure. Lancet. 2017;390(10106):1981-95.

9. Anderson E, Dyke C, Levy JH. Anticoagulation strategies for the management of postoperative atrial fibrillation. Clin Lab Med. 2014;34(3):537-61.

10. January CT, Wann LS, Alpert JS, Calkins H, Cigarroa JE, Cleveland JC, Jr., Conti JB, Ellinor PT, Ezekowitz MD, Field ME, et al. 2014 AHA/ACC/HRS guideline for the management of patients with atrial fibrillation: a report of the American College of Cardiology/American Heart Association Task Force on Practice Guidelines and the Heart Rhythm Society. J Am Coll Cardiol. 2014;64(21):e1-76.

11. Leask A. Potential therapeutic targets for cardiac fibrosis: TGFbeta, angiotensin, endothelin, CCN2, and PDGF, partners in fibroblast activation. Circ Res. 2010;106(11):1675-80.

12. Kakkar R, Lee RT. Intramyocardial fibroblast myocyte communication. Circ Res. 2010;106(1):47-57.

13. Swartz MF, Fink GW, Sarwar MF, Hicks GL, Yu Y, Hu R, Lutz CJ, Taffet SM, Jalife J. Elevated preoperative serum peptides for collagen I and III synthesis result in post-surgical atrial fibrillation. J Am Coll Cardiol. 2012;60(18):1799-806.

14. Xie X, Liu Y, Gao S, Wu B, Hu X, Chen J. Possible involvement of fibrocytes in atrial fibrosis in patients with chronic atrial fibrillation. Circ J. 2014;78(2):338-44.

15. Vandenbroucke RE, Libert C. Is there new hope for therapeutic matrix metalloproteinase inhibition? Nat Rev Drug Discov. 2014;13(12):904-27.

16. Hansen BJ, Zhao J, Fedorov VV. Fibrosis and Atrial Fibrillation: Computerized and Optical Mapping; A View into the Human Atria at Submillimeter Resolution. JACC Clin Electrophysiol. 2017;3(6):531-46.

17. Sohns C, Lemes C, Metzner A, Fink T, Chmelevsky M, Maurer T, Budanova M, Solntsev V, Schulze WHW, Staab W, et al. First-in-Man Analysis of the Relationship Between Electrical Rotors From Noninvasive Panoramic Mapping and Atrial Fibrosis From Magnetic Resonance Imaging in Patients With Persistent Atrial Fibrillation. Circ Arrhythm Electrophysiol. 2017;10(8). 
18. Zykov V, Krekhov A, Bodenschatz E. Fast propagation regions cause self-sustained reentry in excitable media. Proc Natl Acad Sci U S A. 2017;114(6):1281-6.

19. Spinale FG. Myocardial matrix remodeling and the matrix metalloproteinases: influence on cardiac form and function. Physiol Rev. 2007;87(4):1285-342.

20. Volkov AM, Murashov IS, Polonskaya YV, Savchenko SV, Kazanskaya GM, Kliver EE, Ragino YI, AM $\mathrm{CH}$. [Changes of Content of Matrix Metalloproteinases and Their Tissue Expression in Various Types of Atherosclerotic Plaques]. Kardiologiia. 201810):12-8.

21. Xu J, Cui G, Esmailian F, Plunkett M, Marelli D, Ardehali A, Odim J, Laks H, Sen L. Atrial extracellular matrix remodeling and the maintenance of atrial fibrillation. Circulation. 2004;109(3):363-8.

22. Nakano Y, Niida S, Dote K, Takenaka S, Hirao H, Miura F, Ishida M, Shingu T, Sueda T, Yoshizumi M, et al. Matrix metalloproteinase- 9 contributes to human atrial remodeling during atrial fibrillation. $\mathrm{J} \mathrm{Am}$ Coll Cardiol. 2004;43(5):818-25.

23. Ke D, Xu C, Zhang J, Chen L, Lin Y, Lin L, Hu X. Changes in gelatinases expression and activity in human atria during atrial fibrillation. Chinese Journal of Cardiology. 2005;33(2):137-42.

24. De Jong AM, Maass AH, Oberdorf-Maass SU, Van Veldhuisen DJ, Van Gilst WH, Van Gelder IC. Mechanisms of atrial structural changes caused by stretch occurring before and during early atrial fibrillation. Cardiovasc Res. 2011;89(4):754-65.

25. Yabluchanskiy A, Ma Y, lyer RP, Hall ME, Lindsey ML. Matrix metalloproteinase-9: Many shades of function in cardiovascular disease. Physiology (Bethesda). 2013;28(6):391-403.

\section{Figures}

a

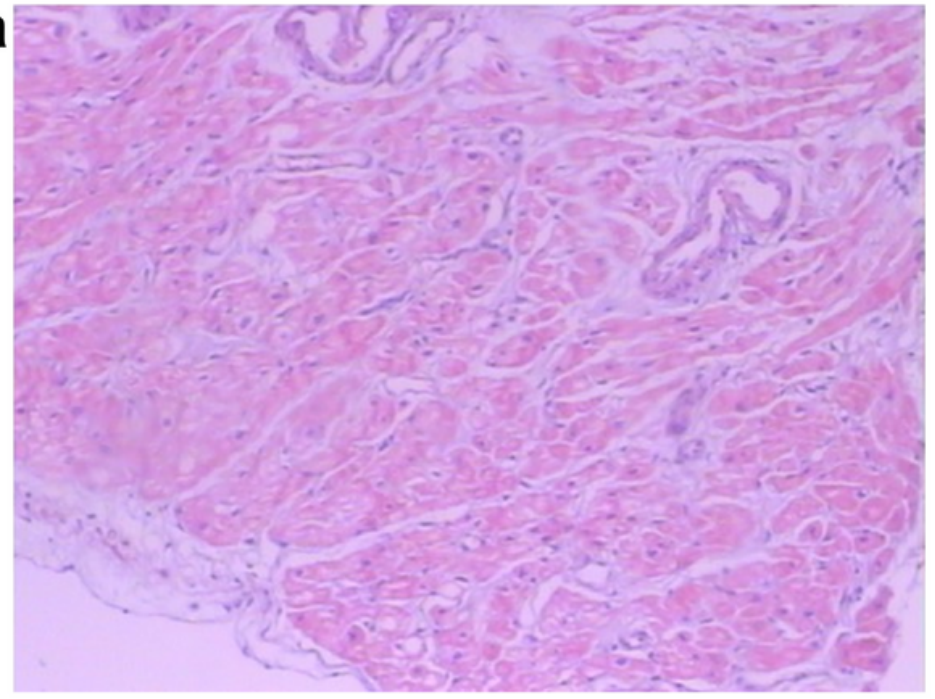

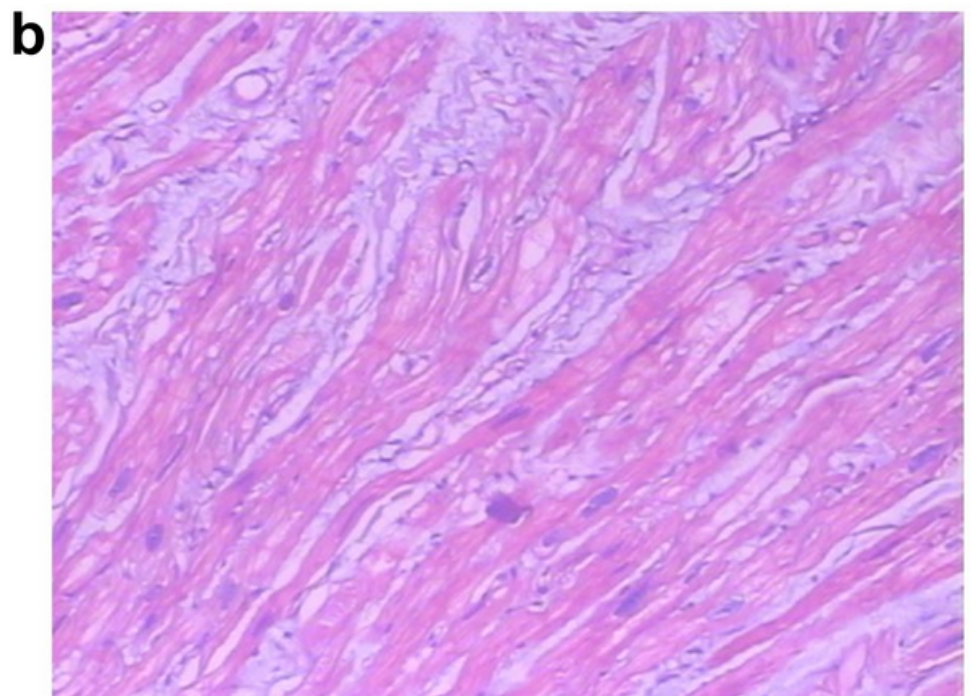

\section{Figure 1}

H\&E staining of the myocardial tissues ( $\times 100)$. a Myocardial tissues of a patient in the SR group, $b$ Myocardial tissues of a patient in the AF group. 
a

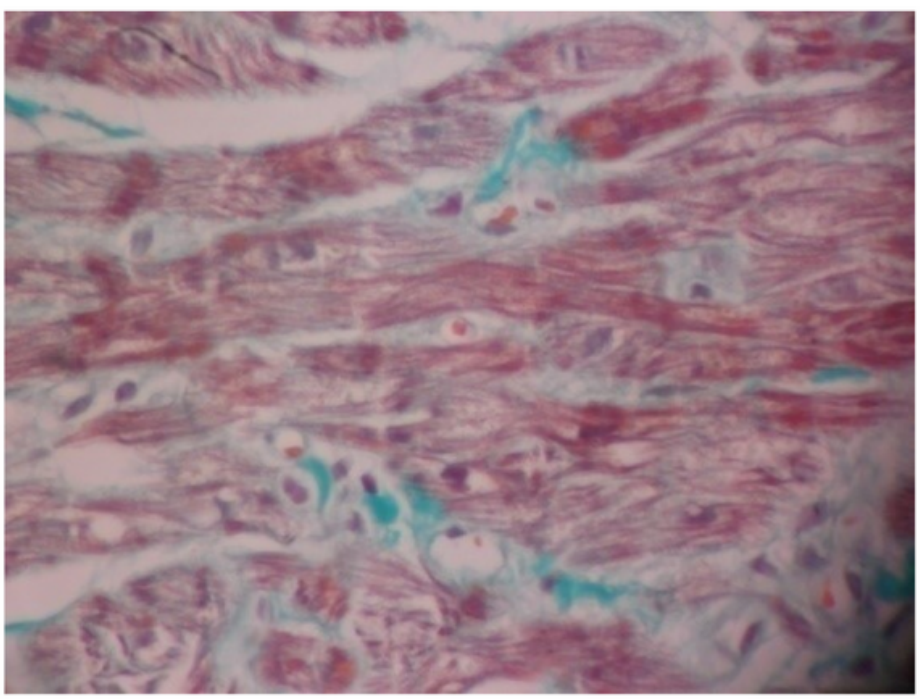

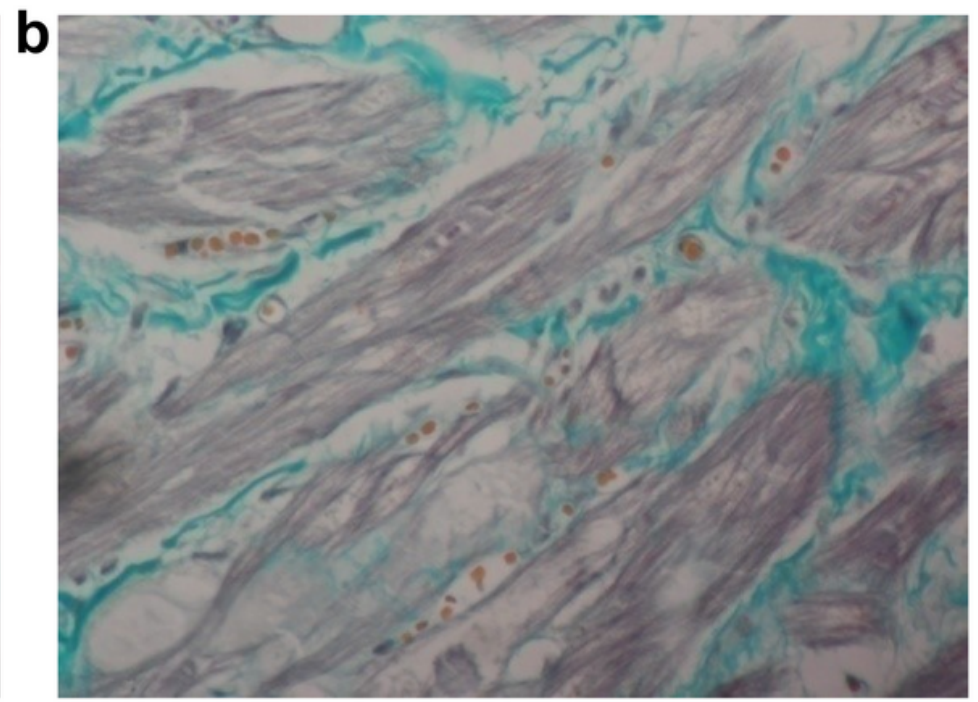

Figure 2

Assessing the expression of collagen fibers in myocardial tissues by Masson staining $(\times 400)$. a Myocardial tissues in SR group, b Myocardial tissues in AF group.

a

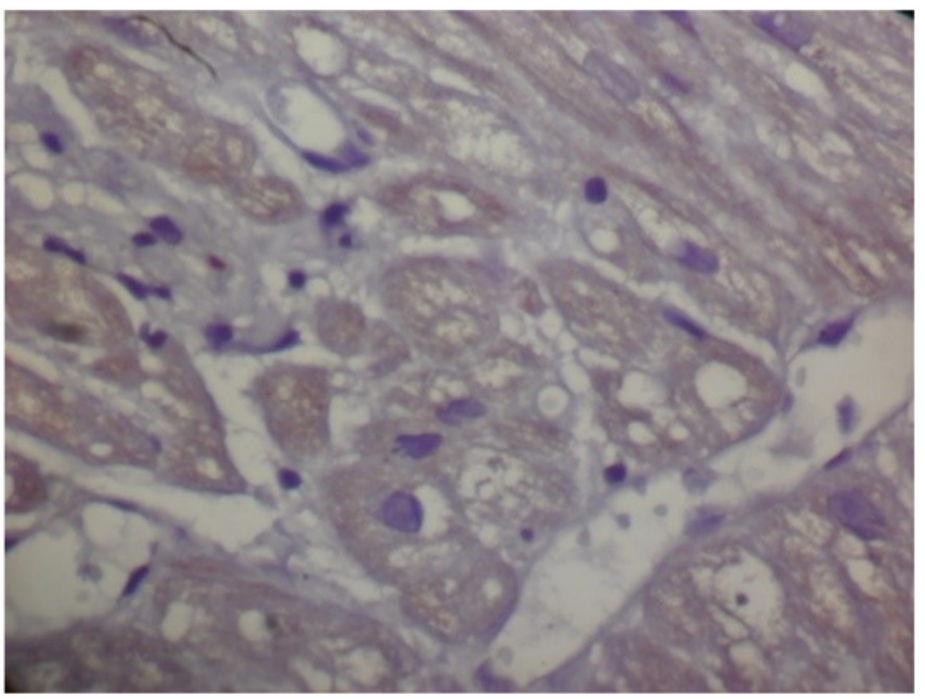

b

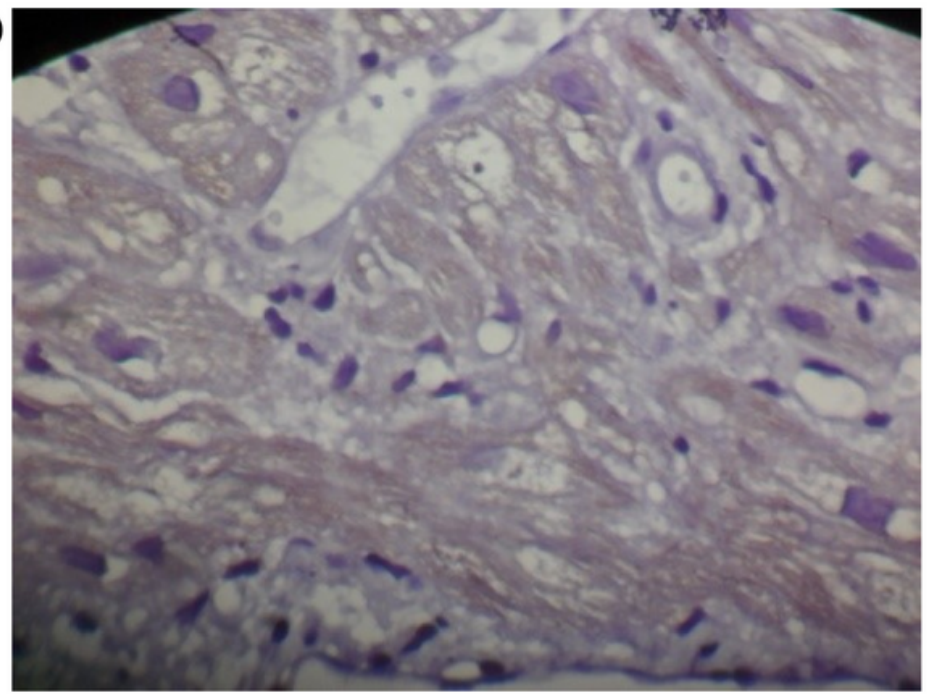

Figure 3

Assessing the expression of MMP-9 in myocardial tissues by immunohistochemistry $(\times 400)$. a Myocardial tissues in SR group, b Myocardial tissues in AF group. 


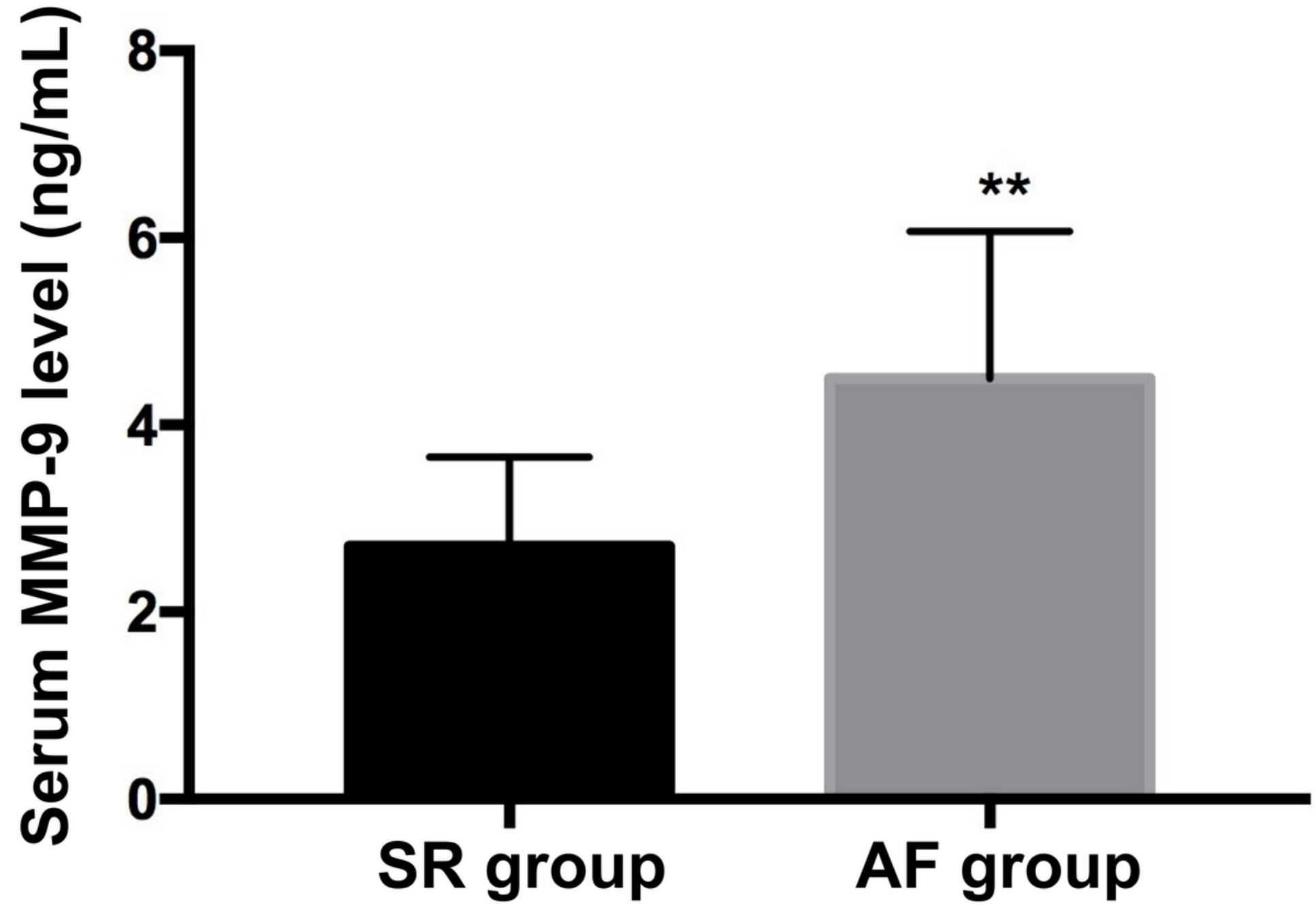

Figure 4

Comparison of MMP-9 levels between the SR and AF groups. ${ }^{*} p<0.05$ compared to the SR group. 


\section{$16.00 \%$}

\section{$14.60 \%$}

$14.00 \%$

$12.00 \%$

우

$10.00 \%$

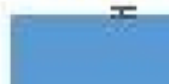

$\stackrel{4}{0}$

$6.00 \%$

$8.00 \%$

$4.00 \%$

$2.00 \%$

$0.00 \%$

\section{AF group}

\section{$8.60 \%$}

Figure 5

Scatter plot of the association between MMP-9 levels and LAD. 


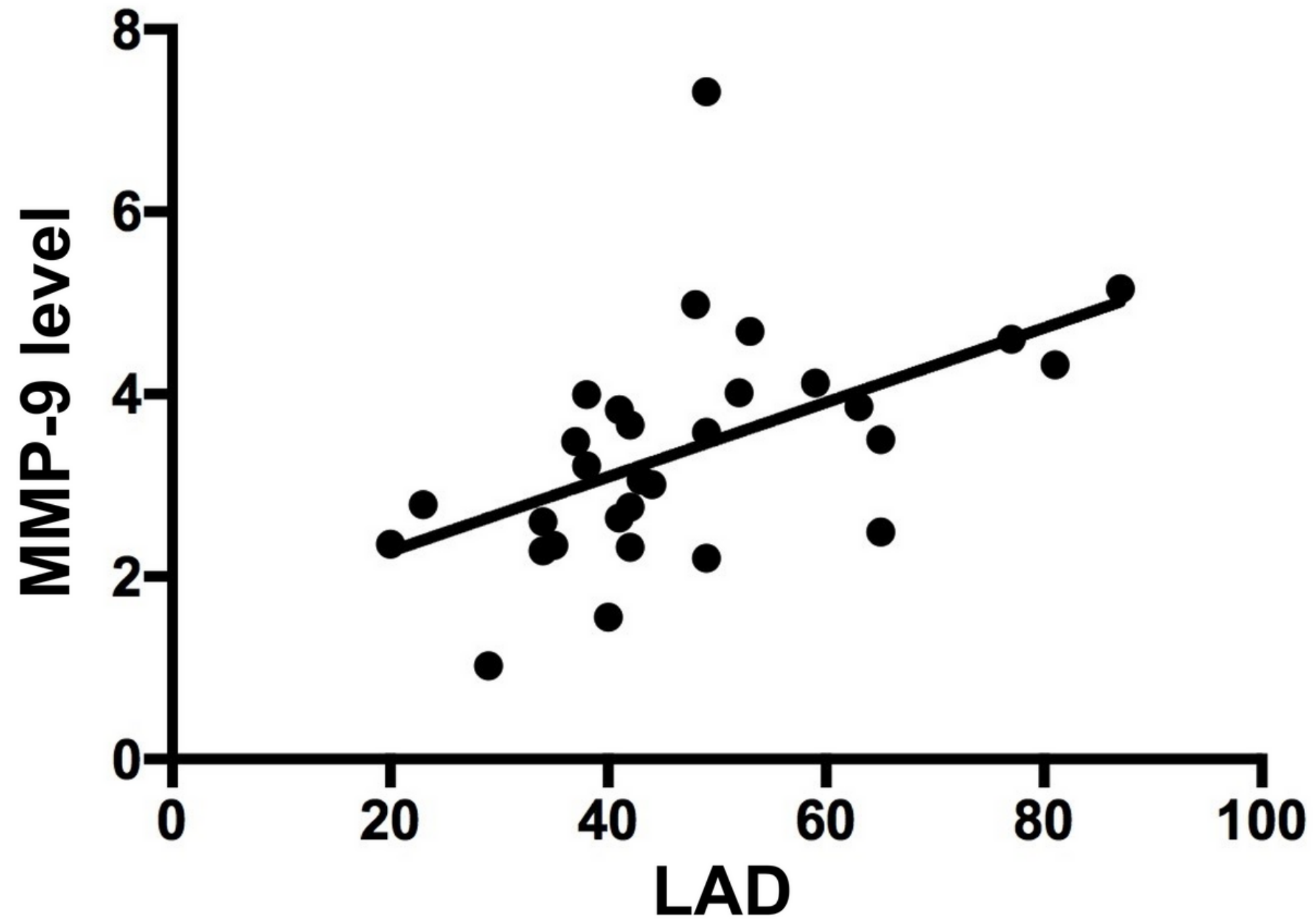

Figure 6

Comparison of CVF between the SR and AF groups. 